\title{
RANDOM FIXED POINT THEOREMS FOR MULTIVALUED NONEXPANSIVE NON-SELF-RANDOM OPERATORS
}

\author{
S. PLUBTIENG AND P. KUMAM
}

Received 8 March 2005; Revised 9 June 2005; Accepted 4 August 2005

Let $(\Omega, \Sigma)$ be a measurable space, with $\Sigma$ a sigma-algebra of subset of $\Omega$, and let $C$ be a nonempty bounded closed convex separable subset of a Banach space $X$, whose characteristic of noncompact convexity is less than $1, K C(X)$ the family of all compact convex subsets of $X$. We prove that a multivalued nonexpansive non-self-random operator $T: \Omega \times C \rightarrow K C(X), 1-\chi$-contractive mapping, satisfying an inwardness condition has a random fixed point.

Copyright (c) 2006 S. Plubtieng and P. Kumam. This is an open access article distributed under the Creative Commons Attribution License, which permits unrestricted use, distribution, and reproduction in any medium, provided the original work is properly cited.

\section{Introduction}

In recent years there have appeared various random fixed point theorems for singlevalued and set-valued random operators; see for example, Itoh [7], Ramírez [9], Tan and Yuan [10], Xu [12,13] Yuan and Yu [15], and references therein.

Ramírez [9] proved the existence of random fixed point theorems for a random nonexpansive operator in the framework of Banach spaces with a characteristic of noncompact convexity $\varepsilon_{\alpha}(X)$ is less than 1 . On the other hand, Domínguez Benavides and Ramírez [4] proved a fixed point theorem for a set-valued nonexpansive self-mapping and 1- $\chi$ contractive mapping in the framework of Banach spaces whose characteristic of noncompact convexity associated to the separation measure of noncompactness $\varepsilon_{\beta}(X)$ is less than 1. Domínguez Benavides and Ramírez [5] proved a fixed point theorem for a multivalued nonexpansive non-self-mapping and $1-\chi$-contractive mapping in the framework of Banach spaces whose characteristic of noncompact convexity associated to the Kuratowski measure of noncompactness $\varepsilon_{\alpha}(X)$ is less than 1 .

The purpose of the present paper is to prove a random fixed point theorem for multivalued nonexpansive non-self-random operators which is $1-\chi$-contractive mapping, in the framework of Banach spaces with characteristic of noncompact convexity associated to the separation measure of noncompactness $\varepsilon_{\beta}(X)$ less than 1 and satisfying an inwardness condition. Our result can also be seen as an extension of [5, Theorem 3.4]. 
2 Random fixed point multivalued nonexpansive non-self-mappings

\section{Preliminaries and notations}

We begin with establishing some preliminaries. By $(\Omega, \Sigma)$ we denote a measurable space with $\Sigma$ a sigma-algebra of subset of $\Omega$. Let $(X, d)$ be a metric space. We denote by $C L(X)$ (resp., $\mathrm{CB}(X), K C(X))$ the family of all nonempty closed (resp., closed bounded, compact convex) subset of $X$, and by $H$ the Hausdorff metric on $\mathrm{CB}(X)$ induced by $d$, that is,

$$
H(A, B)=\max \left\{\sup _{a \in A} d(a, B), \sup _{b \in B} d(b, A)\right\}
$$

for $A, B \in \mathrm{CB}(X)$, where $d(x, E)=\inf \{d(x, y) \mid y \in E\}$ is the distance from $x$ to $E \subset X$.

Let $C$ be a nonempty closed subset of a Banach space $X$. Recall now that a multivalued mapping $T: C \rightarrow 2^{X}$ is said to be upper semicontinuous on $C$ if $\{x \in C: T x \subset V\}$ is open in $C$ whenever $V \subset X$ is open; $T$ is said to be lower semicontinuous if $T^{-1}(V):=\{x \in C$ : $T x \cap V \neq \varnothing$ \} is open in $C$ whenever $V \subset X$ is open; and $T$ is said to be continuous if it is both upper and lower semicontinuous (cf. $[1,2]$ for details). There is another different kind of continuity for multivalued operator: $T: C \rightarrow \mathrm{CB}(X)$ is said to be continuous on $C$ (with respect to the Hausdorff metric $H$ ) if $H\left(T x_{n}, T x\right) \rightarrow 0$ whenever $x_{n} \rightarrow x$. It is not hard to see (see Deimling [2]) that both definitions of continuity are equivalent if $T x$ is compact for every $x \in C$.

If $C$ is a closed convex subset of Banach spaces $X$, then a multivalued mapping $T: C \rightarrow$ $\mathrm{CB}(X)$ is said to be a contraction if there exists a constant $k \in[0,1)$ such that

$$
H(T x, T y) \leq k\|x-y\|, \quad x, y \in C,
$$

and $T$ is said to be nonexpansive if

$$
H(T x, T y) \leq\|x-y\|, \quad x, y \in C
$$

A multivalued operator $T: \Omega \rightarrow 2^{X}$ is called $(\Sigma)$-measurable if, for any open subset $B$ of $X$,

$$
T^{-1}(B)=\{\omega \in \Omega: T(\omega) \cap B \neq \varnothing\}
$$

belongs to $\Sigma$. A mapping $x: \Omega \rightarrow X$ is said to be a measurable selector of a measurable multivalued operator $T: \Omega \rightarrow 2^{X}$ if $x(\cdot)$ is measurable and $x(\omega) \in T(\omega)$ for all $\omega \in \Omega$. An operator $T: \Omega \times C \rightarrow 2^{X}$ is called a random operator if, for each fixed $x \in C$, the operator $T(\cdot, x): \Omega \rightarrow 2^{X}$ is measurable. We will denote by $F(\omega)$ the fixed point set of $T(\omega, \cdot)$, that is,

$$
F(\omega):=\{x \in C: x \in T(\omega, x)\}
$$

Note that if we do not assume the existence of fixed point for the deterministic mapping $T(\omega, \cdot): C \rightarrow 2^{X}, F(\omega)$ may be empty. A measurable operator $x: \Omega \rightarrow C$ is said to be a random fixed point of an operator $T: \Omega \times C \rightarrow 2^{X}$ if $x(\omega) \in T(\omega, x(\omega))$ for all $\omega \in \Omega$. Recall that $T: \Omega \times C \rightarrow 2^{X}$ is continuous if, for each fixed $\omega \in \Omega$, the operator $T:(\omega, \cdot) \rightarrow 2^{X}$ is continuous. 
A random operator $T: \Omega \times C \rightarrow 2^{X}$ is said to be nonexpansive if, for each fixed $\omega \in \Omega$, the map $T:(\omega, \cdot) \rightarrow C$ is nonexpansive.

For later convenience, we list the following results related to the concept of measurability.

Lemma 2.1 (Wagner, cf. [11]). Let $(X, d)$ be a complete separable metric space and $F: \Omega \rightarrow$ $C L(X)$ a measurable map. Then $F$ has a measurable selector.

LeMma 2.2 (Itoh, cf. [7]). Suppose $\left\{T_{n}\right\}$ is a sequence of measurable multivalued operator from $\Omega$ to $\mathrm{CB}(X)$ and $T: \Omega \rightarrow \mathrm{CB}(X)$ is an operator. If, for each $\omega \in \Omega, H\left(T_{n}(\omega), T(\omega)\right) \rightarrow$ 0 , then $T$ is measurable.

Lemma 2.3 (Tan and Yuan, cf. [10]). Let $X$ be a separable metric space and $Y$ a metric space. If $f: \Omega \times X \rightarrow Y$ is measurable in $\omega \in \Omega$ and continuous in $x \in X$, and if $x: \Omega \rightarrow X$ is measurable, then $f(\cdot, x(\cdot)): \Omega \rightarrow Y$ is measurable.

As an easy application of Itoh [7, Proposition 3], we have the following result.

Lemma 2.4. Let $C$ be a closed separable subset of a Banach space $X, T: \Omega \times C \rightarrow C$ a random continuous operator, and $F: \Omega \rightarrow 2^{C}$ a measurable closed-valued operator. Then for any $s>0$, the operator $G: \Omega \rightarrow 2^{C}$ given by

$$
G(\omega)=\{x \in F(\omega):\|x-T(\omega, x)\|<s\}, \quad \omega \in \Omega,
$$

is measurable and so is the operator $\operatorname{cl}\{G(\omega)\}$ of the closure of $G(\omega)$.

Lemma 2.5 (Domínguez Benavidel and Lopez Acedo, cf. [3]). Suppose C is a weakly closed nonempty separable subset of a Banach space $X, F: \Omega \rightarrow 2^{X}$ measurable with weakly compact values, $f: \Omega \times C \rightarrow \mathbb{R}$ measurable, continuous and weakly lower semicontinuous function. Then the marginal function $r: \Omega \rightarrow \mathbb{R}$ defined by

$$
r(\omega):=\inf _{x \in F(\omega)} f(\omega, x)
$$

and the marginal map $R: \Omega \rightarrow X$ defined by

$$
R(\omega):=\{x \in F(\omega): f(\omega, x)=r(\omega)\}
$$

are measurable.

Recall that the Kuratowski and Hausdorff measures of noncompactness of a nonempty bounded subset $B$ of $X$ are, respectively, defined as the numbers

$$
\begin{gathered}
\alpha(B)=\inf \{r>0: B \text { can be covered by finitely many sets of diameter } \leq r\}, \\
\chi(B)=\inf \{r>0: B \text { can be covered by finitely many ball of radius } \leq r\}
\end{gathered}
$$

The separation measure of noncompacness of a nonempty bounded subset $B$ of $X$ is defined by

$$
\beta(B)=\sup \left\{\varepsilon \text { : there exists a sequence }\left\{x_{n}\right\} \text { in } B \text { such that } \operatorname{sep}\left(\left\{x_{n}\right\}\right) \geq \varepsilon\right\} \text {. }
$$


4 Random fixed point multivalued nonexpansive non-self-mappings

Then a multivalued mapping $T: C \rightarrow 2^{X}$ is called $\gamma$-condensing (resp., 1 - $\gamma$-contractive) where $\gamma=\alpha(\cdot)$ or $\chi(\cdot)$ if, for each bounded subset $B$ of $C$ with $\gamma(B)>0$, there holds the inequality

$$
\gamma(T(B))<\gamma(B) \quad(\text { resp., } \gamma(T(B)) \leq \gamma(B)) .
$$

Here $T(B)=\bigcup_{x \in B} T x$. The random operator $T: \Omega \times C \rightarrow 2^{X}$ is said to be $1-\gamma$-contractive if, for each $\omega \in \Omega$, the map $T:(\omega, \cdot) \rightarrow 2^{X}$ is $1-\gamma$-contractive.

Definition 2.1. Let $X$ be a Banach space and $\phi=\alpha, \beta$, or $\chi$. The modulus of noncompact convexity associated to $\phi$ is defined in the following way:

$$
\Delta_{X, \phi}(\varepsilon)=\inf \left\{1-d(0, A): A \subset B_{X} \text { is convex, } \phi(A) \geq \varepsilon\right\},
$$

where $B_{X}$ is the unit ball of $X$.

The characteristic of noncompact convexity of $X$ associated with the measure of noncompactness $\phi$ is defined by

$$
\varepsilon_{\phi}(X)=\sup \left\{\varepsilon \geq 0: \Delta_{X, \phi}(\varepsilon)=0\right\} .
$$

The following relationshops among the different moduli are easy to obtain

$$
\Delta_{X, \alpha}(\varepsilon) \leq \Delta_{X, \beta}(\varepsilon) \leq \Delta_{X, \chi}(\varepsilon)
$$

and consequently

$$
\varepsilon_{\alpha}(X) \geq \varepsilon_{\beta}(X) \geq \varepsilon_{\chi}(X)
$$

When $X$ is a reflexive Banach space, we have some alternative expressions for the moduli of noncompact convexity associated $\beta$ and $\chi$ :

$$
\begin{aligned}
& \Delta_{X, \beta}(\varepsilon)=\inf \left\{1-\|x\|:\left\{x_{n}\right\} \subset B_{X}, x=w-\lim x_{n}, \operatorname{sep}\left(\left\{x_{n}\right\}\right) \geq \varepsilon\right\}, \\
& \Delta_{X, \chi}(\varepsilon)=\inf \left\{1-\|x\|:\left\{x_{n}\right\} \subset B_{X}, x=w-\lim x_{n}, \chi\left(\left\{x_{n}\right\}\right) \geq \varepsilon\right\} .
\end{aligned}
$$

In order to study the fixed point theory for non-self-mappings, we must introduce some terminology for boundary condition. The inward set of $C$ at $x \in C$ is defined by

$$
I_{C}(x):=\{x+\lambda(y-x): \lambda \geq 0, y \in C\} .
$$

Clearly $C \subset I_{C}(x)$ and it is not hard to show that $I_{C}(x)$ is a convex set as $C$ does. A multivalued mapping $T: C \rightarrow 2^{X}\{\varnothing\}$ is said to be inward on $C$ if

$$
T x \subset I_{C}(x) \quad \forall x \in C .
$$

Let $\bar{I}_{C}(x):=x+\{\lambda(z-x): z \in C, \lambda \geq 1\}$. Note that for a convex $C$, we have $\bar{I}_{C}(x)=$ $\overline{I_{C}(x)}$, and $T$ is said to be weakly inward on $C$ if

$$
T x \subset \bar{I}_{C}(x) \quad \forall x \in C .
$$


Let $C$ be a nonempty bounded closed subset of Banach spaces $X$, and $\left\{x_{n}\right\}$ bounded sequence in $X$; we use $r\left(C,\left\{x_{n}\right\}\right)$ and $A\left(C,\left\{x_{n}\right\}\right)$ to denote the asymptotic radius and the asymptotic center of $\left\{x_{n}\right\}$ in $C$, respectively, that is,

$$
\begin{aligned}
& r\left(C,\left\{x_{n}\right\}\right)=\inf \left\{\limsup _{n}\left\|x_{n}-x\right\|: x \in C\right\}, \\
& A\left(C,\left\{x_{n}\right\}\right)=\left\{x \in C: \limsup _{n}\left\|x_{n}-x\right\|=r\left(C,\left\{x_{n}\right\}\right)\right\} .
\end{aligned}
$$

If $D$ is a bounded subset of $X$, the Chebyshev radius of $D$ relative to $C$ is defined by

$$
r_{C}(D):=\inf \{\sup \{\|x-y\|: y \in D\}: x \in C\}
$$

Obviously, the convexity of $C$ implies that $A\left(C,\left\{x_{n}\right\}\right)$ is convex. Notice that $A\left(C,\left\{x_{n}\right\}\right)$ is a nonempty weakly compact set if $C$ is weakly compact, or $C$ is a closed convex subset of a reflexive Banach spaces $X$.

Let $\left\{x_{n}\right\}$ and $C$ be nonempty bounded closed subsets of Banach spaces $X$. Then $\left\{x_{n}\right\}$ is called regular with respect to $C$ if $r\left(C,\left\{x_{n}\right\}\right)=r\left(C,\left\{x_{n_{i}}\right\}\right)$ for all subsequences $\left\{x_{n_{i}}\right\}$ of $\left\{x_{n}\right\}$; while $\left\{x_{n}\right\}$ is called asymptotically uniform with respect to $C$ if $A\left(C,\left\{x_{n}\right\}\right)=$ $A\left(C,\left\{x_{n_{i}}\right\}\right)$ for all subsequences $\left\{x_{n_{i}}\right\}$ of $\left\{x_{n}\right\}$.

Lemma 2.6 (Goebel [6] and Lim [8]). Let $\left\{x_{n}\right\}$ and $C$ be as above. Then we have the following:

(i) there always exists a subsequence of $\left\{x_{n}\right\}$ which is regular with respect to $C$;

(ii) if $C$ is separable, then $\left\{x_{n}\right\}$ contains a subsequence which is asymptotically uniform with respect to $C$.

Moreover, we also need the following lemma.

Lemma 2.7 (Domínguez Benavides and Ramírez, cf. [4, Theorem 3.4]). Let $C$ be a closed convex subset of reflexive Banach spaces $X$, and let $x_{n}$ be a bounded sequence in $C$ which is regular with respect to $C$. Then

$$
r_{C}\left(A\left(C, x_{n}\right)\right) \leq\left(1-\Delta_{X, \beta}\left(1^{-}\right)\right) r\left(C,\left\{x_{n}\right\}\right) .
$$

Moreover, if $X$ satisfies the nonstrict Opial condition, then

$$
r_{C}\left(A\left(C, x_{n}\right)\right) \leq\left(1-\Delta_{X, \chi}\left(1^{-}\right)\right) r\left(C,\left\{x_{n}\right\}\right) .
$$

Lemma 2.8 (Domínguez Benavides and Ramírez, cf. [5, Theorem 3.2]). Let $C$ be a closed convex subset of a reflexive Banach space $X$, and let $\left\{x_{\beta}: \beta \in D\right\}$ be a bounded ultranet. Then

$$
r_{C}\left(A\left(C, x_{\beta}\right)\right) \leq\left(1-\Delta_{X, \alpha}\left(1^{-}\right)\right) r\left(C,\left\{x_{\beta}\right\}\right)
$$

The following result are now basic in the fixed point theorem for multivalued mappings. 
6 Random fixed point multivalued nonexpansive non-self-mappings

Lemma 2.9 (Deimling, cf. [2]). Let $X$ be a Banach space and $\varnothing \neq D \subset X$ be closed bounded convex. Let $F: D \rightarrow 2^{X}$ be upper semicontinuous $\gamma$-condensing with closed convex values, where $\gamma(\cdot)=\alpha(\cdot)$ or $\chi(\cdot)$. If $F x \cap \overline{I_{D}(x)} \neq \varnothing$ for all $x \in C$, then $F$ has a fixed point. (Here $I_{D}(x)$ is called the inward set at $x$ defined by $I_{D}(x):=\{x+\lambda(y-x): \lambda \geq 0, y \in D\}$.)

\section{The result}

In order to prove our first result, we need the following lemma which is proved along the proof of Kirk-Massa theorem as it appears in [14].

LeMma 3.1. Let $C$ be a nonempty closed bounded convex separable subset of a Banach space $X . T: C \rightarrow K C(X)$ is nonexpansive such that $T(C)$ is a bounded set which satisfies $T x \subset$ $I_{C}(x), \forall x \in C,\left\{x_{n}\right\}$ is a sequence in $C$ such that $\lim _{n} d\left(x_{n}, T x_{n}\right)=0$. Then there exists $a$ subsequence $\left\{z_{n}\right\}$ of $\left\{x_{n}\right\}$ such that $T x \cap I_{A}(x) \neq \varnothing, \forall x \in A:=A\left(C,\left\{z_{n}\right\}\right)$.

Lemma 3.1 is part (more or less) of the proof of [5, Theorem 3.4].

The next result states the main result of this work.

Theorem 3.2. Let $C$ be a nonempty closed bounded convex separable subset of Banach spaces $X$ such that $\epsilon_{\beta}(X)<1$, and $T: \Omega \times C \rightarrow K C(X)$ a multivalued nonexpansive random operator and 1- $\chi$-contractive mapping, such that for each $\omega \in \Omega, T(\omega, C)$ is a bounded set, which satisfies the inwardness condition, that is, for each $\omega \in \Omega, T(\omega, x) \subset I_{C}(x), \forall x \in C$.

Then $T$ has a random fixed point.

Proof. Fix $x_{0} \in C$, and consider the measurable function $x_{0}(\omega) \equiv x_{0}$. For each $n \geq 1$, define $T_{n}(\omega, \cdot): C \rightarrow K C(X)$ by

$$
T_{n}(\omega, x)=\frac{1}{n} x_{0}(\omega)+\left(\frac{n-1}{n}\right) T(\omega, x), \quad \forall x \in C .
$$

Then $T_{n}(\omega, \cdot)$ is a multivalued contraction and $T_{n}(\omega, x) \subset I_{C}(x), \forall x \in C$. Hence each $T_{n}$ has a fixed point $z_{n}(\omega) \in C$. It is easily seen that $d\left(z_{n}(\omega), T\left(\omega, z_{n}(\omega)\right)\right) \leq(1 / n) \operatorname{diam} C \rightarrow 0$ as $n \rightarrow \infty$. Thus the set

$$
F_{n}(\omega)=\left\{x \in C: d(x, T(\omega, x)) \leq \frac{1}{n} \operatorname{diam} C\right\}
$$

is nonempty closed and convex. Furthermore, by Lemma 2.4, each $F_{n}$ is measurable. Then, by Lemma 2.1, each $F_{n}$ admits a measurable selector $x_{n}(\omega)$ such that

$$
d\left(x_{n}(\omega), T\left(\omega, x_{n}(\omega)\right)\right) \leq \frac{1}{n} \operatorname{diam} C \longrightarrow 0 \quad \text { as } n \longrightarrow \infty
$$

Define a function $f: \Omega \times C \rightarrow \mathbb{R}^{+}:=[0, \infty)$ by

$$
f(\omega, x)=\limsup _{n}\left\|x_{n}(\omega)-x\right\|, \quad x \in C .
$$

By Lemma 2.3 , it is easily seen that $f(\cdot, x)$ is measurable and $f(\omega, \cdot)$ is continuous and convex, therefore it is a weakly lower semicontinuous function. Note that; condition 
$\varepsilon_{\beta}(X)<1$ implies reflexivity (see $[1]$ ) and so $C$ is a weakly compact. Hence, by Lemma 2.5, the marginal functions

$$
\begin{gathered}
r(\omega):=\inf _{x \in C} f(\omega, x), \\
A(\omega):=\{x \in C: f(\omega, x)=r(\omega)\}
\end{gathered}
$$

are measurable. It is clearly that $A(\omega)$ is a weakly compact convex subset of $C$. For any $\omega \in \Omega$, we may assume that the sequence $\left\{x_{n}(\omega)\right\}$ is regular with respect to $C$. Note that $A(\omega)=A\left(C,\left\{x_{n}(\omega)\right\}\right)$, and $r(\omega)=r\left(C,\left\{x_{n}(\omega)\right\}\right)$. We can apply inequality (2.22) in Lemma 2.7 to obtain

$$
r_{C}(A(\omega)) \leq \lambda r\left(C,\left\{x_{n}(\omega)\right\}\right),
$$

where $\lambda=1-\Delta_{X, \beta}\left(1^{-}\right)<1$, since $\varepsilon_{\beta}(X)<1$.

For each $\omega \in \Omega$ and $n \geq 1$, we define the multivalued contraction $T_{n}^{1}(\omega, \cdot): A(\omega) \rightarrow$ $K C(X)$ by

$$
T_{n}^{1}(\omega, x)=\frac{1}{n} x_{1}(\omega)+\left(\frac{n-1}{n}\right) T(\omega, x)
$$

for each $x \in C$. By Lemma 3.1, we note that $T(\omega, x) \cap I_{A(\omega)}(x) \neq \varnothing, \forall x \in A(\omega)$. Since $I_{A(\omega)}(x)$ is convex, it follows that $T_{n}^{1}(\omega, \cdot)$ satisfies the boundary condition, that is,

$$
T_{n}^{1}(\omega, x) \cap I_{A(\omega)}(x) \neq \varnothing, \quad \forall x \in A(\omega) .
$$

Since $T_{n}^{1}(\omega, \cdot)$ is $1-\chi$-contractive mapping, it follows by [4, page 382] that $T_{n}^{1}(\omega, \cdot)$ is $\chi$-condensing. Hence, by Lemma $2.9, T_{n}^{1}(\omega, \cdot)$ has a fixed point $z_{n}^{1}(\omega) \in A(\omega)$, that is, $F(\omega) \cap A(\omega) \neq \varnothing$. Also it is easily seen that

$$
\operatorname{dist}\left(z_{n}^{1}(\omega), T\left(\omega, z_{n}^{1}(\omega)\right)\right) \leq \frac{1}{n} \operatorname{diam} C \longrightarrow 0 \quad \text { as } n \longrightarrow \infty .
$$

Thus $F_{n}^{1}(\omega):=\{x \in A(\omega): d(x, T(\omega, x)) \leq(1 / n)$ diam $C\}$ is nonempty closed and convex for each $n \geq 1$. By Lemma 2.4, each $F_{n}^{1}$ is measurable. Hence, by Lemma 2.1, we can choose $x_{n}^{1}$ a measurable selector of $F_{n}^{1}$. Thus we have $x_{n}^{1}(\omega) \in A(\omega)$ and $d\left(x_{n}^{1}(\omega), T(\omega\right.$, $\left.\left.x_{n}^{1}(\omega)\right)\right) \rightarrow 0$ as $n \rightarrow \infty$. Consider the function $f_{2}: \Omega \times C \rightarrow \mathbb{R}^{+}$defined by

$$
f_{2}(\omega, x)=\limsup _{n}\left\|x_{n}^{1}(\omega)-x\right\|, \quad \forall \omega \in \Omega
$$

As above, $f_{2}$ is a measurable function and weakly lower semicontunuous function. Then the marginal functions

$$
\begin{gathered}
r_{2}(\omega):=\inf _{x \in A(\omega)} f_{2}(\omega, x), \\
A^{1}(\omega):=\left\{x \in A(\omega): f_{2}(\omega, x)=r_{2}(\omega)\right\}
\end{gathered}
$$

are measurable. Since $A^{1}(\omega)=A\left(A(\omega),\left\{x_{n}^{1}(\omega)\right\}\right)$, it follows that $A^{1}(\omega)$ is a weakly compact and convex. Moreover, we also note that $r_{2}(\omega)=r\left(A(\omega),\left\{x_{n}^{1}(\omega)\right\}\right)$. Again reasoning 
8 Random fixed point multivalued nonexpansive non-self-mappings

as above, for any $\omega \in \Omega$, we can assume that the sequence $\left\{x_{n}^{1}(\omega)\right\}$ is regular with respect to $A^{1}(\omega)$. Moreover, we proceed as above using Lemmas 3.1 and 2.7 to obtain that

$$
\begin{gathered}
T(\omega, x(\omega)) \cap I_{A^{1}}(x(\omega)) \neq \varnothing \quad \forall x(\omega) \in A^{1}=A\left(A(\omega),\left\{x_{n}^{1}(\omega)\right\}\right), \\
r_{C}\left(A^{1}\right) \leq \lambda r\left(A(\omega),\left\{x_{n}^{1}(\omega)\right\}\right) \leq \lambda r_{C}(A(\omega)) .
\end{gathered}
$$

By induction, for each $m \geq 1$, we take a sequence $\left\{x_{n}^{m}(\omega)\right\}_{n} \subseteq A^{m-1}$ such that $r_{C}\left(A^{m}\right) \leq$ $\lambda^{m} r_{C}(A(\omega))$ and $\lim _{n} d\left(x_{n}^{m}(\omega), T\left(\omega, x_{n}^{m}(\omega)\right)\right)=0$ for each fixed $\omega \in \Omega$, where $A^{m}:=A(C$, $\left.\left\{x_{n}^{m}(\omega)\right\}\right)$. Since $\operatorname{diam} R_{m}(\omega) \leq 2 r_{C}\left(R_{m}(\omega)\right)$ and $\lambda<1$, it follows that $\lim _{m \rightarrow \infty} \operatorname{diam} R_{m}(\omega)=$ 0 . Note that $\left\{R_{m}(\omega)\right\}$ is a descending sequence of weakly compact subset of $C$ for each $\omega \in \Omega$. Thus we have $\cap_{m} R_{m}(\omega)=\{z(\omega)\}$ for some $z(\omega) \in C$. Furthermore, we see that

$$
H\left(R_{m}(\omega),\{z(\omega)\}\right) \leq \operatorname{diam} R_{m}(\omega) \longrightarrow 0 \quad \text { as } n \longrightarrow+\infty .
$$

Therefore, by Lemma 2.2, $z(\omega)$ is measurable. Finally, we will show that $z(\omega)$ is a fixed point of $T$. Indeed, for each $m \geq 1$, we have

$$
\begin{aligned}
d(z(\omega), T(\omega, z(\omega))) \leq & \left\|z(\omega)-x_{n}^{m}(\omega)\right\|+d\left(x_{n}^{m}(\omega), T\left(\omega, x_{n}^{m}(\omega)\right)\right) \\
& +H\left(T\left(\omega, x_{n}^{m}(\omega)\right), T(\omega, z(\omega))\right) \\
\leq & 2\left\|z(\omega)-x_{n}^{m}(\omega)\right\|+d\left(x_{n}^{m}(\omega), T\left(\omega, x_{n}^{m}(\omega)\right)\right) \\
\leq & 2 \operatorname{diam} R_{m}(\omega)+d\left(x_{n}^{m}(\omega), T\left(\omega, x_{n}^{m}(\omega)\right)\right) .
\end{aligned}
$$

Taking the upper limit as $n \rightarrow \infty$,

$$
d(z(\omega), T(\omega, z(\omega))) \leq 2 \operatorname{diam} R_{m}(\omega) .
$$

Finally, taking limit in $m$ in both sides, we obtain $z(\omega) \in T(\omega, z(\omega))$.

Theorem 3.3. Let $C$ be a nonempty closed bounded convex separable subset of Banach spaces $X$ such that $\epsilon_{\alpha}(X)<1$, and $T: \Omega \times C \rightarrow K C(X)$ a multivalued nonexpansive random operator and 1-X-contractive nonexpansive mapping, such that for each $\omega \in \Omega, T(\omega, C)$ is a bounded set, which satisfies the inwardness condition, that is, for each $\omega \in \Omega, T(\omega, x) \subset$ $I_{C}(x), \forall x \in C$.

Then $T$ has a random fixed point.

Proof. Following from Theorem 3.2 and using Lemma 2.8.

Corollary 3.4. Let $C$ be a nonempty closed bounded convex subset of Banach spaces $X$ such that $\epsilon_{\beta}(X)<1$. If $T: C \rightarrow K C(X)$ is a multivalued nonexpansive and $1-\chi$-contractive nonexpansive mapping, such that $T(C)$ is a bounded set, which satisfies the inwardness condition, that is, for each $T x \subset I_{C}(x), \forall x \in C$.

Then $T$ has a fixed point.

Corollary 3.5 (Domínguez Benavides and Ramírez, cf. [5, Theorem 3.4]). Let X be Banach spaces such that $\varepsilon_{\alpha}(X)<1$, and $C$ a nonempty closed bounded convex subset of $X$. If $T: C \rightarrow K C(X)$ is nonexpansive and 1- $\chi$-contractive nonexpansive mapping, such that $T(C)$ is a bounded set, which satisfies $T x \subset I_{C}(x) \forall x \in C$, then $T$ has a fixed point. 


\section{Acknowledgment}

The first author would like to thank The Thailand Research Fund for financial support.

\section{References}

[1] J. M. Ayerbe Toledano, T. Domínguez Benavides, and G. López Acedo, Measures of Noncompactness in Metric Fixed Point Theory, Operator Theory: Advances and Applications, vol. 99, Birkhäuser, Basel, 1997.

[2] K. Deimling, Nonlinear Functional Analysis, Springer, Berlin, 1974.

[3] T. Domínguez Benavides, G. López Acedo, and H.-K. Xu, Random fixed points of set-valued operators, Proceedings of the American Mathematical Society 124 (1996), no. 3, 831-838.

[4] T. Domínguez Benavides and P. Lorenzo Ramírez, Fixed-point theorems for multivalued nonexpansive mappings without uniform convexity, Abstract and Applied Analysis 2003 (2003), no. 6, $375-386$.

[5] __ Fixed point theorems for multivalued nonexpansive mappings satisfying inwardness conditions, Journal of Mathematical Analysis and Applications 291 (2004), no. 1, 100-108.

[6] K. Goebel, On a fixed point theorem for multivalued nonexpansive mappings, Annales Universitatis Mariae Curie-Skłodowska 29 (1975), 69-72.

[7] S. Itoh, A random fixed point theorem for a multivalued contraction mapping, Pacific Journal of Mathematics 68 (1977), no. 1, 85-90.

[8] T. C. Lim, Remarks on some fixed point theorems, Proceedings of the American Mathematical Society 60 (1976), 179-182.

[9] P. Lorenzo Ramírez, Some random fixed point theorems for nonlinear mappings, Nonlinear Analysis 50 (2002), no. 2, 265-274.

[10] K.-K. Tan and X.-Z. Yuan, Some random fixed point theorems, Fixed Point Theory and Applications (Halifax, NS, 1991) (K.-K. Tan, ed.), World Scientific, New Jersey, 1992, pp. 334-345.

[11] D.-H. Wagner, Survey of measurable selection theorems, SIAM Journal on Control and Optimization 15 (1977), no. 5, 859-903.

[12] H.-K. Xu, Some random fixed point theorems for condensing and nonexpansive operators, Proceedings of the American Mathematical Society 110 (1990), no. 2, 395-400.

[13] _ Random fixed point theorems for nonlinear uniformly Lipschitzian mappings, Nonlinear Analysis 26 (1996), no. 7, 1301-1311.

[14] _ Multivalued nonexpansive mappings in Banach spaces, Nonlinear Analysis 43 (2001), no. 6, 693-706.

[15] X.-Z. Yuan and J. Yu, Random fixed point theorems for nonself mappings, Nonlinear Analysis 26 (1996), no. 6, 1097-1102.

S. Plubtieng: Department of Mathematics, Faculty of Science, Naresuan University,

Phitsanulok 65000, Thailand

E-mail address: somyotp@nu.ac.th

P. Kumam: Department of Mathematics, Faculty of Science, King Mongkut's University of Technology Thonburi, Bangkok 10140, Thailand

E-mail address: pooom.kum@kmutt.ac.th 


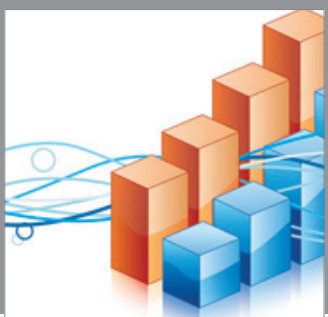

Advances in

Operations Research

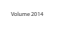

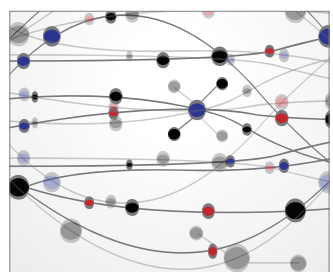

\section{The Scientific} World Journal
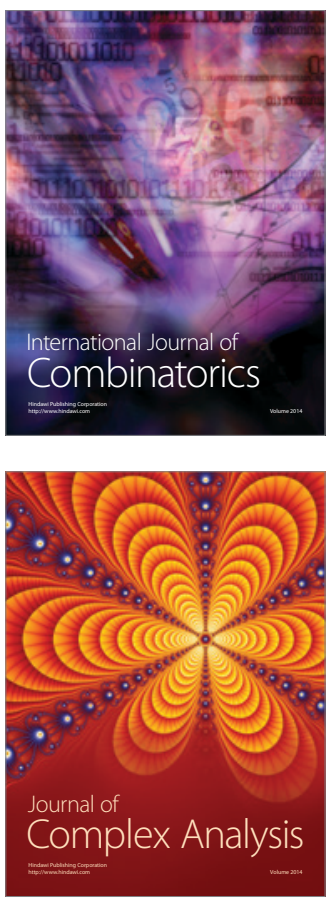

International Journal of

Mathematics and

Mathematical

Sciences
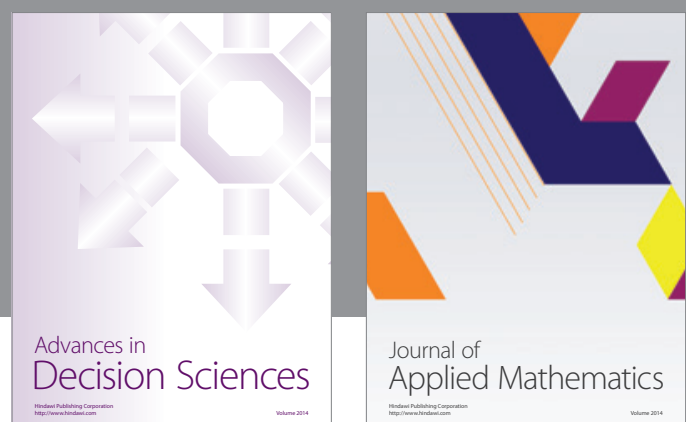

Journal of

Applied Mathematics
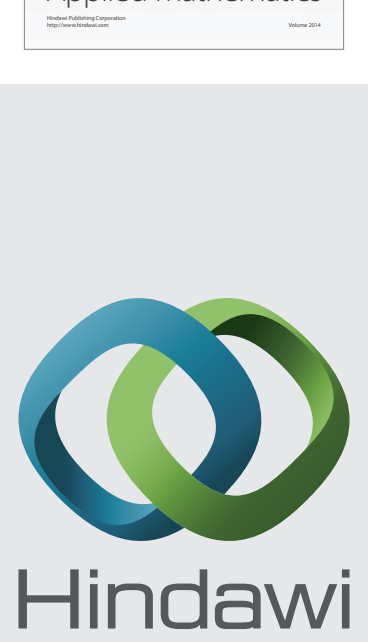

Submit your manuscripts at http://www.hindawi.com
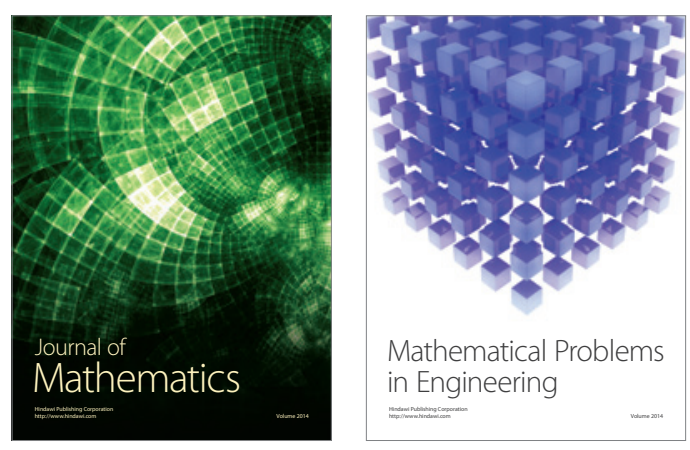

Mathematical Problems in Engineering
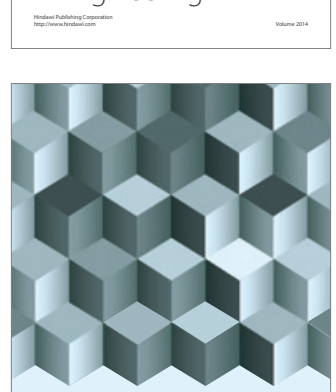

Journal of

Function Spaces
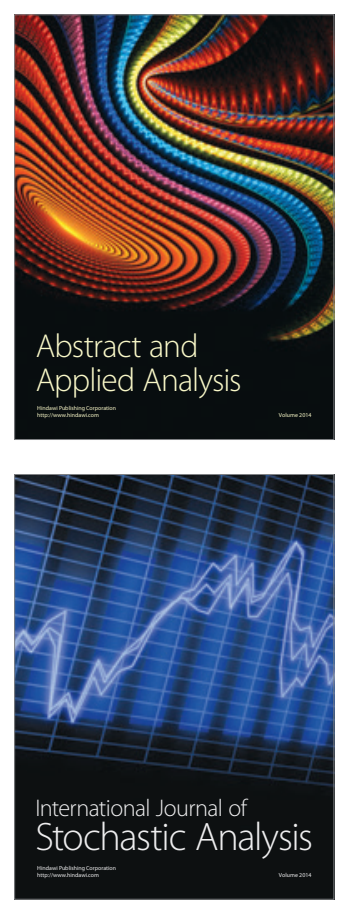

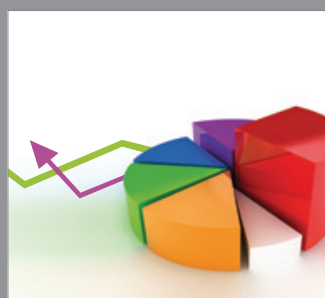

ournal of

Probability and Statistics

Promensencen
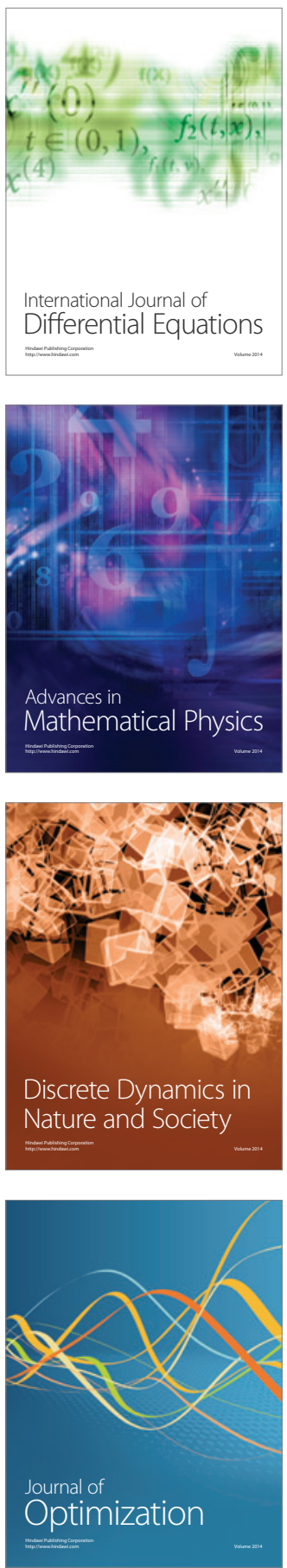